\title{
Uso de Facebook en Educación Superior: análisis de la relación entre participación y calificaciones
}

\author{
Isabel María Solano-Fernández \\ imsolano@um.es \\ Universidad de Murcia, España \\ Pedro Antonio García-Tudela \\ pedroantonio.garcia4@um.es \\ Universidad de Murcia, España \\ María del Mar Sánchez-Vera \\ mmarsanchez@um.es \\ Universidad de Murcia, España
}

\begin{abstract}
Resumen
Las redes sociales en educación superior presentan un enorme potencial didáctico, en el marco de un proceso didáctico que ponga en valor la comunicación entre los agentes educativos, la colaboración como metodología de enseñanza y la participación activa como medio para lograr un aprendizaje más significativo y contextualizado. En el marco de una experiencia de innovación educativa basada en el uso de Facebook en el Grado en Educación Infantil de la Universidad de Murcia (España se analiza la relación entre la participación del alumnado en esta red social y sus calificaciones en la asignatura "Medios, Materiales y TIC". Se ha empleado una metodología cuantitativa con un diseño de investigación ex post facto a partir de las calificaciones obtenidas por los estudiantes $(n=172)$, con las que se ha llevado a cabo un análisis de correlaciones múltiple. Los resultados ponen de manifiesto las diferencias significativas existentes en las calificaciones obtenidas por los tres grupos de la asignatura, así como en la relación existente entre la participación en la red social social Facebook y la calificación final de la asignatura, evidenciándose que los alumnos que obtienen mejores calificaciones, son los que más han participado en la red social. La experiencia llevada a cabo evidencia las potencialidades de Facebook como recurso complementario como parte de un proceso reflexivo y planificado. Asimismo, plantea la necesidad de completar investigaciones como la presente con planteamientos de investigación de corte que permita dar cuenta de las diferencias en función de la metodología empleada por el profesorado.-
\end{abstract}

\section{Palabras clave}

Tecnología Educativa; Enseñanza Superior; Participación; Redes Sociales; Evaluación 


\title{
The use of Facebook in Higher Education: analysis of the relationship between participation and subject marks
}

\author{
Isabel María Solano-Fernández \\ imsolano@um.es \\ University of Murcia, Spain \\ Pedro Antonio García-Tudela \\ pedroantonio.garcia4@um.es \\ University of Murcia, Spain \\ María del Mar Sánchez-Vera \\ mmar.sanchez@um.es \\ University of Murcia, Spain
}

\begin{abstract}
Social networks in Higher Education have enormous educational potential, within the framework of a teaching process that values communication between educational agents, collaboration as a teaching methodology and active participation as a means of achieving more meaningful and contextualised learning. Within the framework of an experience of educational innovation based on the use of Facebook in early childhood education Degree at University of Murcia (Spain). From the implementation, the relationship between the participation of students on this social network and their mark in the subject "Medios, Materiales y TIC" is analyzed. For this purpose, a quantitative methodology is used, with an ex post facto research design based on the marks obtained by the students $(n=172)$, with which a multiple correlation analysis has been carried out. The results show significant differences in the grades obtained by the three groups of the subject, as well as in the relationship between participation in the social network Facebook and the final grade of the subject, showing that the students who obtain the best grades are those who have participated most in the social network. The experience carried out shows the potential of Facebook as a complementary resource as part of a reflective and planned process. Likewise, it raises the need to complete research such as this with cutting-edge research approaches that allow the differences in terms of the methodology used by the teaching staff to be accounted for.
\end{abstract}

\section{Keywords}

Educational technology; Higher Education; Participation; Social Networks; assessment. 


\section{Introducción}

La incorporación de las tecnologías en la enseñanza sigue presentando grandes desafíos, independientemente del nivel educativo (Gamito, et al., 2019). El debate tradicional de la Tecnología Educativa en torno a la innovación tecnológica y la innovación pedagógica sigue presente en nuestro sistema educativo, y la didáctica sigue ofreciendo una gran oportunidad para dar entidad a los estudios e investigaciones que surgen acerca de cómo podemos integrar las tecnologías en las aulas (Cabero \& Aguaded, 2007).

Una de las variables determinantes en el proceso educativo es el alumnado que encontramos en las aulas es, ya que son el reflejo de la sociedad en la que vivimos y de sus cambios sociales y culturales. Por lo tanto, al profesorado universitario se le plantea el reto de dialogar entre la sociedad cambiante y las competencias que el alumnado debe desarrollar para enfrentarse de manera competente y así, convertirse en motor de cambio. En este sentido, Imbernón (2016) realiza una lúcida reflexión sobre los retos del profesorado ante una sociedad en cambio, destacando el papel clave de este para que las nuevas generaciones construyan un mundo mejor y se conviertan en motor de cambio:

La mejora de la profesión docente tiene que ayudar al profesorado a construir y reconstruir el desarrollo de la infancia y la juventud para que lleguen al máximo de sus capacidades y puedan vivir mejor. $Y$ muchos lo hacen con la ilusión que las nuevas generaciones que ocupan las aulas puedan construir un mundo mejor. Esta es la verdadera finalidad de la educación: no tanto reproducir el que se ha hecho, aunque sea necesario conocerlo, como sobre todo avanzarse al futuro (p.123).

En el marco de la digitalización, se han hecho diversas aportaciones que se centran en analizar el tipo de alumnado que tenemos, producto de la incorporación de las tecnologías de manera habitual en el día a día. La denominación «nativo digital» fue desarrollada por Prensky (2001) para referirse a todas las personas que han nacido y se han formado utilizando información digital en diferentes formatos. Aunque los estudiantes continúan siendo representados por dicha etiqueta (Uğur \& Koç, 2019), el propio Prensky ha señalado que el término no siempre se ha interpretado de forma adecuada, alejándose del carácter metafórico que él le atribuyó originalmente (Prensky \& González, 2018). Las discrepancias en el término han llevado a autores como White (2011) a considerar que la terminología de Prensky ha quedado superada con el paso de los años, y que realmente no podemos hablar de nativos e inmigrantes digitales, sino de residentes y visitantes digitales. El residente es la persona que «vive» un porcentaje de su vida en línea, es la persona que desarrolla parte de su identidad en la red y ésta forma un entorno más en el que comunicarse o hacer otras acciones (White \& Le Cornu, 2011). En cualquier caso, de una u otra forma, lo cierto es que la Universidad del siglo XXI debe responder a las demandas de aprendizaje de todos los estudiantes, los cuales poseen una alta influencia del uso de la red y diferentes dispositivos electrónicos (Calzado et al., 2018).

A pesar de las percepciones que pueden existir respecto a que las aulas cuentan con «alumnos digitales», resulta interesante conocer el trabajo de Prendes y Román (2017), en el cual se expone que el alumnado universitario considera la clase presencial como el elemento más importante de su formación, así como que los materiales de lectura tradicionales están mejor valorados que el uso de otras aplicaciones como blogs o redes sociales. Estos resultados pueden deberse al hecho de que, a pesar de estar inmersos en la sociedad digital, el alumnado universitario se ve condicionado por su experiencia académica, todavía anclada en la clase magistral y recursos no tecnológicos.

No existe una iniciativa óptima que sea extensible a toda la población universitaria, ya que incluir la tecnología educativa en el aula ha de consistir en la adaptación y la selección de los recursos y estrategias más adecuadas para las características del alumnado (Cabero, et al., 2007), entre otros aspectos. No obstante, los estudiantes aprovechan la inmediatez y las diferentes oportunidades 
que las redes sociales brindan para trabajar colaborativamente de manera extraescolar (GutiérrezPorlán, et al., 2018). Es por ello que diversos autores han puesto de manifiesto que las redes sociales son óptimas y potencialmente beneficiosas para su implementación en el contexto educativo superior (De Haro, 2009; González-Pérez, 2018; Gutiérrez-Porlán, et al., 2018; Lytras, 2018; Marín-Díaz, Cabero-Almenera, 2019).

De manera opuesta a la última afirmación, y desde una perspectiva discente, determinados estudiantes consideran que los aspectos académicos han de estar separados de las herramientas de ocio, ya que estas pueden causar distracciones, ausencia de responsabilidad docente, etc. (Vivar-Zurita, et al., 2011).

Sin embargo, cualquier planificación didáctica ha de tener en cuenta qué tipo de alumnado recibe en el aula y cuáles son sus necesidades, para así, desde la responsabilidad científica y pedagógica, se consiga articular una docencia que sea útil y relevante. Entender que el alumnado es el protagonista de la acción educativa implica que se contemple un análisis de qué necesidades tiene y se oriente la actividad docente a partir de sus conocimientos y experiencias previas.

El uso de Facebook resulta especialmente interesante por el potencial que las redes sociales pueden brindar a la educación universitaria, hecho destacado por algunos autores, ya que aportan un nuevo entorno y, con ello, un soporte comunicativo (Túñez, et al., 2015); pueden mejorar el impacto emocional y cubrir las necesidades de comunicación (Rocha, et al., 2015), y además, mejorar el proceso de aprendizaje y la calidad educativa (Arteaga-Sánchez et al, 2019; Cornejo \& Lariza, 2016; Chugh \& Ruhi, 2018; Davidovitch, \& Belichenko, 2018).

Existen diversas redes sociales que explícitamente se dedican a un uso educativo (Interuniversidades, Clipit, Docsicity, Edmodo, etc.). Sin embargo, una de las redes sociales mejor valoradas en cuanto a su potencial en educación superior es Facebook (Calzado, et al., 2018: Martínez \& Ferraz, 2016), aunque no siempre se ha utilizado asiduamente y de la mejor forma posible (Arteaga et al., 2014). Twitter, también se valora como un entorno adecuado para su uso en educación universitaria, aunque algunos estudiantes expresan su descontento por la sobrecarga de información que ofrece, la motivación extrínseca que genera, entre otras razones (Adams et al., 2018).

Focalizando en el objeto de estudio, son numerosos los casos en los que Facebook se ha utilizado complementariamente a las clases presenciales de diversos estudios universitarios (Nicolai, et al., 2017; Zanon, et al., 2018), entre ellos, algunos impartidos en las Facultades de Educación (DíazLázaro et al., 2017; Martínez \& Ferraz, 2016).

Facebook puede utilizarse de distintas formas. En algunos casos, el docente es el único administrador de la cuenta en la que progresivamente publica información a través de vídeos tutoriales (Zuñe \& Rosas, 2016), o de la misma manera, solo hay un perfil único de la asignatura controlado por los docentes de una asignatura (Serrat, 2015); también se utiliza como una agenda en red a partir de la que concertar reuniones, crear avisos como cambios de horario o aulas, etc. (López de la Madrid et al., 2015), o bien, con un uso similar al desarrollado en este trabajo, es decir, la publicación de información complementaria a los contenidos trabajados en el aula (Cabeza, et al., 2014; Díaz-Lázaro et al., 2017).

Para Chugh y Ruhi (2018), son muchas las posibilidades que ofrece Facebook para la Educación Superior, entre las que destaca el desarrollo de actividades de aprendizaje pedagógicas usando Facebook como plataforma, como portafolio electrónico que promueva la colaboración, investigación y discusión, para fomentar la participación y motivar hacia el aprendizaje. No olvidemos que Facebook sigue siendo la red social con más usuarios activos, unos 2.700 millones en octubre de 2020 (Facebook, 2020), y aunque otras aplicaciones como Instagram, Snapchap, Pinterest o whatapp se están mostrando eficaces para la colaboración en procesos de enseñanza en 
línea (Manca, 2020), Facebook, todavía hoy, presenta un enorme potencial en la Educación Superior por cuanto supone para la mejora del sentimiento de comunidad, el fomento del compromiso educativo por parte de los estudiantes, y para la mejora de los resultados educativos, como ponen de manifiesto las investigaciones de Arteaga-Sánchez et al (2019) y Chugh y Ruhi (2018), entre otros.

Un adecuado diseño de la propuesta y un desarrollo apropiado de la misma, beneficiará buenas prácticas que favorecerán el alcance de los objetivos preestablecidos. De manera general, algunos de los logros señalados están relacionados con la mejora de la comunicación docente-discentes y entre estudiantes (Shraim, 2014; Chugh \& Ruhi, 2018); solución de dudas sobre los contenidos impartidos (López de la Madrid, et al., 2017); desarrollo del pensamiento crítico (Castro \& González-Palta, 2016); incremento de la participación (Cunha et al., 2016; Thai et al, 2019) motivación hacia la temática estudiada (Viviar-Zurita et al., 2011) y fortalecimiento del proceso de enseñanza-aprendizaje y del trabajo colaborativo (Abúndez et al., 2015).

Existen algunas desventajas indicadas por el alumnado en torno al uso de Facebook, tales como ser una herramienta de ocio que interrumpe su tarea académica y que tampoco garantiza privacidad y seguridad en la red (Iglesias-García \& González, 2014; Vivar-Zurita, et al., 2011). Por su parte, en la revisión bibliométrica realizada por Chugh y Ruhi (2018) se puso de manifiesto que algunos de los desafíos y limitaciones de Facebook como recurso para la enseñanza hacen referencia a la falta de dominio por parte del profesorado, el comportamiento inactivo de parte del alumnado, la falta del uso de un lenguaje académico, la preocupación por los problemas técnicos y de privacidad y la discriminación motivada por la brecha generacional. A pesar de ello, y mayoritariamente, los estudiantes valoran positivamente el uso didáctico que se hace de Facebook en la Educación Superior (Arteaga-Sánchez, 2019; Shraim, 2014).

Con el objetivo de prevenir malas prácticas, existen propuestas de mejora que generalmente, se resumen en ofrecer una formación permanente al profesorado sobre cómo utilizar dicha red social en el contexto educativo y establecer un diseño pautado y consensuado con los estudiantes sobre la temporalización y modo de uso (López de la Madrid, et al., 2015 y 2017). También Chugh y Ruhi (2018), a la luz de las investigaciones analizadas, proponen unas orientaciones para usar Facebook en la Educación Superior.

Para analizar lo que sucede en estos entornos virtuales, han surgido muchas herramientas que se denominan analíticas de aprendizaje que permiten conocer qué hacen los alumnos en estos entornos. También han cobrado auge las Social Learning Analytics o analíticas del aprendizaje social, es decir, la recopilación, medición, análisis e informe referente a los estudiantes y su interacción en un contexto digital (Siemens \& Gasevic, 2012). Dichas analíticas procesan de manera cuantitativa y cualitativa una gran cantidad de datos para ofrecer resultados conclusivos, o también favorecer la adaptación de las publicaciones según el interés y las demandas del alumnado (Díaz-Lázaro et al., 2017).

Teniendo en cuenta la información reflejada hasta el momento, y siendo conscientes del enorme potencial que tiene Facebook para promover la participación del alumnado con el resto de estudiantes y con el profesorado, y de los efectos positivos que esto puede tener para la consecución de los objetivos académicos, el problema de investigación del presente trabajo se expresa en la siguiente cuestión: ¿cómo influye una propuesta basada en el uso de la red social Facebook en los Grados de Infantil de la Universidad de Murcia en relación con las calificaciones finales obtenidas en la asignatura? 


\section{Metodología}

El presente estudio ex post-facto se define como un estudio correlacional, puesto que el principal interés es determinar las relaciones existentes entre las variables más significativas de la propuesta implementada. Concretamente, estamos ante un estudio de correlación múltiple, ya que están implicadas más de dos variables de manera simultánea (Mateo, 2004).

\section{a. Propuesta de innovación}

En el marco de la asignatura obligatoria «Medios, Materiales y TIC para la enseñanza en Educación Infantil» (6 ECTS) impartida en la Universidad de Murcia, se diseñó e implementó una propuesta de innovación a través de la red Social Facebook entre enero y mayo de 2019.

En la experiencia participaron los 3 grupos del Grado en Educación Infantil de la Universidad de Murcia, ya que un $10 \%$ de la calificación final de la asignatura dependía de la participación del alumnado en Facebook. La distribución de la calificación final de la asignatura se estructuró en un $60 \%$ de prácticas, que incluye el diseño de varias propuestas educativas con recursos didácticos, y un $40 \%$ correspondiente a una prueba de evaluación oral.

Para facilitar la participación y llevar un mayor control de la misma se crearon 3 grupos privados en la mencionada red social, correspondientes con los 3 grupos del Grado mencionado. En cada grupo de Facebook existía la figura del administrador y moderador, pudiendo ambos participar de manera libre cada semana con la publicación de entradas, y por supuesto, con comentarios y reacciones a las publicaciones de los alumnos. Con la publicación de entradas se aportaba información complementaria a la tratada en clase, para que así, el alumnado comentase sus ideas o conocimientos al respecto.

Además de la interacción con las publicaciones del moderador, el alumnado podía participar de diferentes formas con su grupo de Facebook: escribiendo una entrada con información de interés (vídeos o vínculos de buenas prácticas de algún recurso tratado en clase; reflexiones personales sobre la temática abordada, etc.), comentando las publicaciones de otros compañeros o del moderador, compartiendo las prácticas de grupo a través de un link a sus blogs o también, reaccionando («me gusta», «me sorprende», etc.) a otras entradas.

La calificación final de cada estudiante en relación al uso de Facebook fue extraída teniendo en cuenta la actividad en dicha red social. Para este fin, se establecieron diferentes criterios atendiendo al número y a la calidad de las interacciones en el grupo privado. Por lo tanto, publicar una entrada no tenía el mismo valor que comentar o, simplemente, reaccionar ante una publicación. De manera más específica, la puntuación que se estableció para cada acción fue la siguiente: publicaciones $(.65 \%)$, comentarios a publicaciones $(.35 \%)$ y reacciones (no puntúa).

\section{b. Participantes}

Tal y como se ha reflejado anteriormente, todos los estudiantes que cursaron la asignatura «Medios, Materiales y TIC para la enseñanza en Educación Infantil» participaron en la propuesta de innovación desarrollada con Facebook. No obstante, la muestra invitada fue de 190 alumnos, y la muestra productora de datos quedó finalmente constituida en 172. Aunque el tamaño de la muestra garantiza la representatividad, puesto que con un nivel de confianza del $95 \%$, el margen de error es mínimo $(2.31 \%)$, es importante indicar que con los resultados de esta investigación no pretenden ser generalizables.

A continuación, se expone un conciso análisis descriptivo atendiendo a variables sociodemográficas para favorecer el posterior entendimiento del análisis de resultados. 
En primer lugar, la participación de estudiantes por cada uno de los grupos es bastante similar (grupo $1[n=69]$, grupo $2[n=65]$ y grupo $3[n=56]$ ), ya que la diferencia más significativa es de 13 alumnos. Concretamente, entre el grupo 1 y el 3.

En relación al sexo de la muestra, sí que se obtienen unas diferencias representativas, ya que los hombres representan el $3.7 \%$, mientras que las mujeres el $96.3 \%$. Por lo tanto, el sexo es un factor que se descarta para el análisis.

El último factor que se presenta es la edad, para ello se ha relacionado con el grupo al que pertenece. La edad mínima son 19 años, mientras que la máxima son 51 años. No obstante, la mediana del grupo 1 y 2 se sitúa en 20 años y la correspondiente al grupo 3 son 22 años. En la Tabla 1 se exponen los principales datos descriptivos según la edad y el grupo. La relativa homogenidad de la muestra en cuanto a la variable edad nos lleva también a no considerarla para el estudio.

\begin{tabular}{lllllllll}
\hline & N & Mín. & Q1 & Mediana & Media & Sd & Q3 & Máx. \\
\hline Grupo 1 & 69 & 19 & 20 & 20 & 22.70 & 6.60 & 22 & 51 \\
Grupo 2 & 65 & 19 & 20 & 20 & 21.14 & 2.42 & 22 & 31 \\
Grupo 3 & 56 & 21 & 21 & 22 & 23.07 & 4.14 & 23 & 47 \\
\hline
\end{tabular}

Tabla 1. Datos descriptivos de la edad según el grupo

\section{c. Análisis de datos}

Los datos recogidos han sido analizados utilizando el software $R$ versión 3.6.1 ( $R$ Core Team, 2019). El problema de investigación planteado ha sido abordado desde el análisis de matrices de correlación de las diferentes variables tenidas en cuenta, es decir, actas de junio y julio, exámenes de junio y julio, prácticas de la asignatura, comentarios en Facebook y publicaciones en Facebook. Para determinar la normalidad de dicha prueba, se ha tenido en cuenta el teorema central del límite, el cual estudia el comportamiento de la suma de variables, asegurando su convergencia hacia una distribución normal (Alvarado \& Batanero, 2008).

Complementariamente, se analizan de manera aislada las variables señaladas en el párrafo anterior, para así, favorecer el entendimiento de las matrices de correlación y extraer unas conclusiones significativas en relación a la pregunta de investigación planteada. Previamente a realizar una ANOVA de una vía con la correlación de Welch, de cada variables se han comprobado los supuestos de normalidad (prueba de Shapiro-Wilk) y homocedasticidad (prueba de Bartlett).

Los diferentes resultados descriptivos sobre una variable según el grupo al que pertenece son reflejados en forma de tabla y diagrama boxplot. En cambio, para interpretar los resultados mostrados en el gráfico matriz de correlaciones se debe tener en cuenta si es una correlación inversa o directa, y por lo tanto, el grado de efecto extraído.

\section{Resultados}

En primer lugar, se considera de interés explicitar los resultados extraídos según las actas publicadas, ya que una décima parte de la calificación final depende de la actividad en Facebook. A continuación, en la Figura 1 se aprecia el boxplot del acta de junio. 


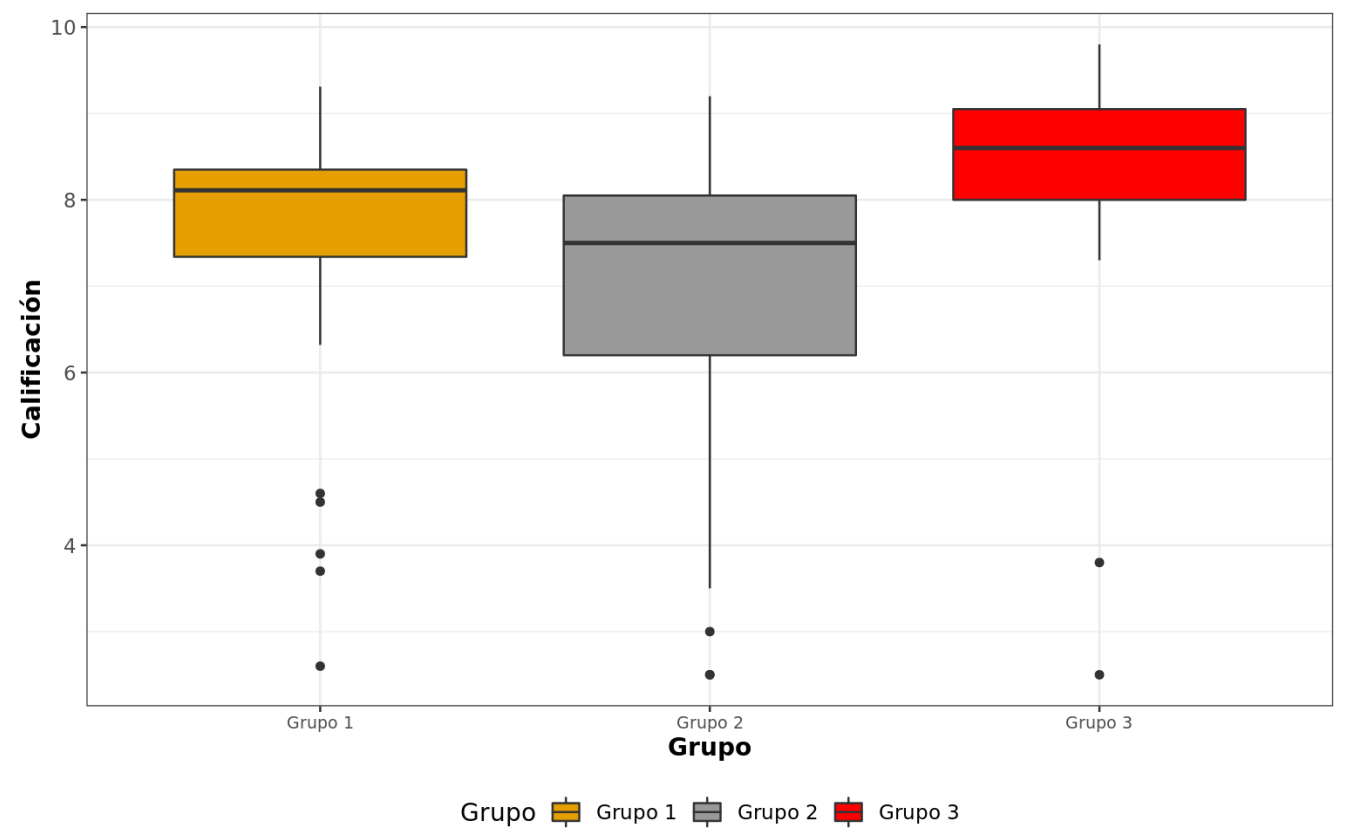

Figura. 1. Diagrama de boxplot de la calificación del acta de junio

La nota mínima (suspenso 2.5) es compartida por el grupo 1 y 2 , mientras que la máxima (sobresaliente 10) le pertenece al grupo 2 . Sin embargo, la mediana más alta le corresponde al grupo 3 , la cual se sitúa en un notable 8.3.

Al aplicar la prueba de normalidad Shapiro-Wilk se obtiene que no hay normalidad en algunos datos, pero se puede suponer por tener más de 30 casos en cada grupo según el teorema central del límite (Tobón y Bedoya, 2017). Igualmente, se obtiene que no hay homocedasticidad. Por lo tanto, se decide aplicar el análisis de ANOVA de una vía con la correlación de Welch. El resultado pone de manifiesto que $\mathrm{F}(2,94.09)=9.4612, \mathrm{p}=2 \times 10-4$, es decir, el $\mathrm{p}$-valor es menor que .05 , por lo que se rechaza la hipótesis nula y se confirma que hay diferencias significativas en las calificaciones finales de junio según el grupo.

Al aplicar una prueba de comparación por pares con desviaciones estándar agrupadas se obtiene que existen diferencias significativas entre la calificación del grupo 1 y el grupo 3 , y entre el grupo 2 y el grupo 3.

Seguidamente, en la Figura 2 se expone el boxplot correspondiente al acta de julio según el grupo. 


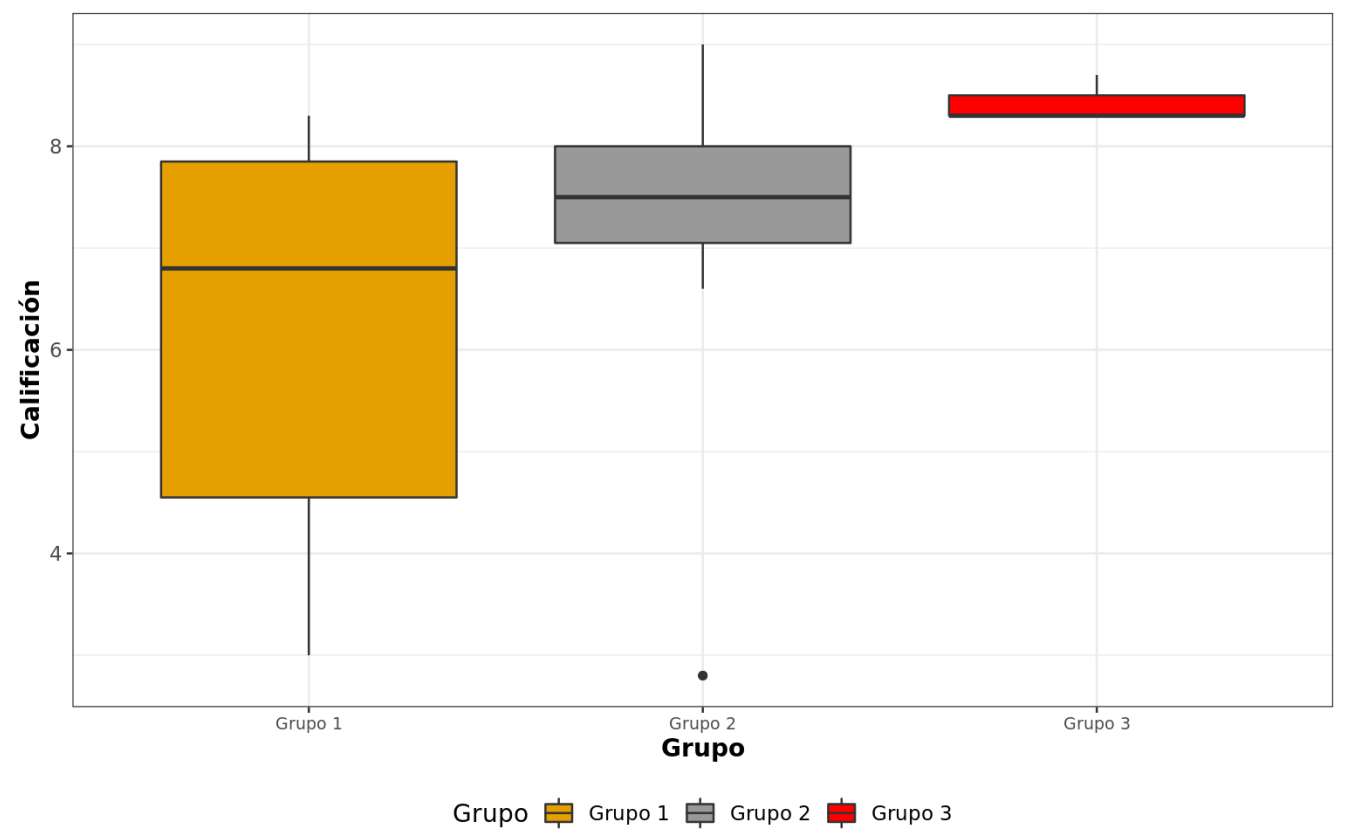

Figura. 2. Diagrama de boxplot de la calificación del acta de julio

En este caso, la calificación mínima asciende ligeramente respecto al acta de junio (suspenso 2,8), y le pertenece al grupo 2. En cambio, existe un descenso de un punto en la máxima (sobresaliente 9), la cual le corresponde al mismo grupo. Al igual que anteriormente, la mediana más alta está representada por un 8.43 del grupo 3 .

En relación a la prueba de normalidad, también se obtiene la misma conclusión que en la variable acta de junio. En cambio, al no haber más de 30 estudiantes en los grupos no se puede aplicar el análisis ANOVA de una vía y se opta por el test no paramétrico de $\mathrm{K}$ de Kruskal-Wallis (LópezSoto, 2013). En el mismo se obtiene $\mathrm{X} 2(2)=6.3896, \mathrm{p}=.041$, al ser $\mathrm{p}$-valor menor a .05 , se ha de rechazar la hipótesis nula de que no hay diferencias significativas en la variable acta julio según el grupo.

Con el fin de determinar las diferencias entre grupos en el acta de julio se hace uso de la prueba de sumas de rangos Wilcoxon. Esta expone que las únicas diferencias significativas que hay en la calificación son entre el grupo 2 y el grupo 3 .

Una vez expuestos los resultados relativos a las calificaciones de la muestra, a continuación se analiza la interacción (comentarios y publicaciones) en Facebook de los diferentes grupos.

En primer lugar, en relación a los comentarios en Facebook, en la Tabla 2 se presentan los datos descriptivos pertinentes para determinar las diferencias entre los grupos participantes.

\begin{tabular}{lllllllll}
\hline & N & Mín. & Q1 & Mediana & Media & Sd & Q3 & Máx. \\
\hline Grupo 1 & 69 & 0 & 0 & 2 & 5.36 & 11.43 & 6 & 83 \\
Grupo 2 & 65 & 0 & 0 & 2 & 3.72 & 5.50 & 4 & 26 \\
Grupo 3 & 56 & 0 & 0 & 0 & .18 & .58 & 0 & 3 \\
\hline
\end{tabular}

Tabla 2. Interacción en Facebook según grupo 
En los tres grupos coincide el valor mínimo, el cual es 0, que es asignado a los alumnos que no han participado en la red social, o han tenido una participación casi nula. Por el contrario, el máximo está representado por el número 83, que en este caso se corresponde con un estudiante del grupo 1 que ha realizado 83 comentarios en publicaciones de su grupo de Facebook. Este dato contrasta con el máximo de comentarios realizados en el grupo 3, que es de 3. Esto nos hace focalizar nuestra atención en las posibles diferencias de participación de los grupos 1 y 2 con el 3 . De hecho, los dos primeros obtienen una mediana similar, concretamente, con un valor 2, lo cual evidencia una desproporción bastante llamativa en cuanto a la realización de comentarios respecto al grupo 3 , cuya mediana es 0 . De igual forma, el dato de la desviación estándar de los grupos 1 (SD = $11.43)$ y $2(S D=5.50)$ también reflejan esta situación de escasa participación del grupo 3.

Al igual que anteriormente, se realiza la prueba Shapiro Wilk y se obtiene que no hay normalidad en los datos, pero la misma se puede suponer por tener más de 30 casos en cada grupo según el teorema central del límite. Por otro lado, al aplicar la prueba de Barlett, se obtiene que no existe homocedasticidad. Por lo tanto, se aplica el ANOVA de una vía con la corrección de Welch. El resultado obtenido es $F(2,89.32)=20.1264, p=0.000$. Dado que el $p$-valor es menor a .05 y se ha de rechazar la hipótesis nula de que no hay diferencias significativas en la variable comentarios en Facebook según el grupo. La prueba de comparación por parejas expone que las principales diferencias se sitúan entre el grupo 1 y el grupo 3, y entre el grupo 2 y el grupo 3. Por tanto, a través de esta prueba confirmamos que existen diferencias en la participación, por medio de comentarios, entre los grupos.

La última variable que se analiza de manera aislada previamente a exponer las matrices de correlación es la relativa a publicaciones en Facebook según grupo, es decir, las entradas que se escriben en el grupo (véase Tabla 3).

\begin{tabular}{lllllllll}
\hline & N & Mín. & Q1 & Mediana & Media & Sd & Q3 & Máx. \\
\hline Grupo 1 & 69 & 0 & 2.00 & 4 & 5.55 & 5.07 & 8 & 25 \\
Grupo 2 & 65 & 0 & 2.00 & 2 & 4.23 & 4.49 & 5 & 28 \\
Grupo 3 & 56 & 0 & 2.75 & 3 & 2.66 & 1.67 & 4 & 7 \\
\hline
\end{tabular}

Tabla 3. Publicaciones en Facebook según grupo

De la misma manera que en la variable de comentarios en Facebook, ahora los valores mínimos también son coincidentes en los tres grupos. No sucede lo mismo que anteriormente con los valores máximos, ya que en este caso, excepto el grupo 3 que presenta un valor 7 , los grupos 1 y 3 muestran un elevado número de publicaciones en la red social por parte de algún o algunos alumnos, siendo de 25 y 28 publicaciones respectivamente.

De la misma forma, la mediana no es coincidente, pero tampoco con un valor 0 como en el grupo 3 en la variable de comentarios en Facebook. En este caso, existe un rango que oscila de 2 a 4.

Los datos referentes a las publicaciones en los correspondientes grupos tampoco presentan normalidad y homocedasticidad, aunque la primera se puede suponer al igual que en otros casos previos. Por consiguiente, se aplica el ANOVA de una vía con la corrección de Welch y se obtiene $\mathrm{F}$ $(2,107.65)=11.9571, p=.000$ De esta forma, se ha de rechazar la hipótesis nula, y por tanto, constatamos nuevamente que hay diferencia entre los tres grupos en lo que respecta a las 
publicaciones, poniéndose de relieve que el grupo 1 y 2 han realizado más publicaciones en la red social que los alumnos del grupo 3.

Tal y como se ha ido señalando a lo largo del trabajo, la calificación final de la asignatura, analizada a través de las actas correspondientes con las convocatorias de junio y julio) depende directamente de la suma ponderada de los resultados extraídos del examen sumativo, las actividades prácticas de la asignatura y la interacción en Facebook. Para analizar esta correlación múltiple se han generado unas matrices que exponen las relaciones existentes entre las diferentes variables (Figura 3). Para interpretar los datos de una manera adecuada se han de tener en cuenta tanto el tipo de correlación (inversa o directa) y el tamaño del efecto, el cual puede oscilar de -1 a 1 . Dicha escala puede ser interpretada atendiendo a diferentes denominaciones, por ejemplo el valor 0 es nulo, entre 0 y +1 es positiva e imperfecta, etc. (Mateo, 2004). No obstante, en este caso se consideran los siguientes efectos:

$\mathrm{s}<|.1|$ : efecto despreciable.

$|.1|<\mathrm{s}<|.3|$ : efecto pequeño.

$|.3|<\mathrm{s}<|.5|$ : efecto mediano.

$s>|.5|$ : efecto grande.

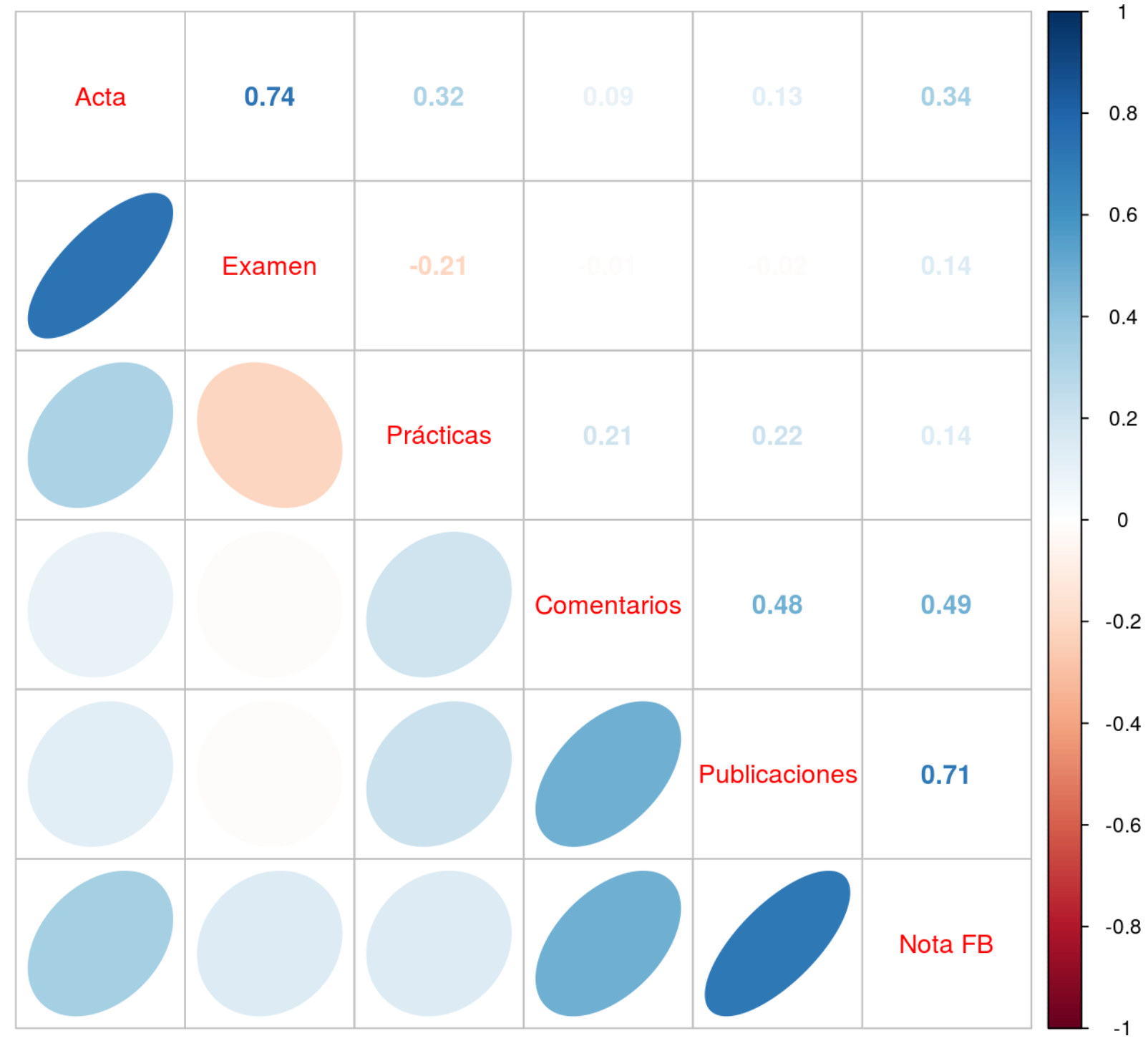


Figura. 3. Matrices de correlación de las variables

Fuente: elaboración propia

Teniendo en cuenta que el efecto grande es cuando $s>|.5|$, tan solo se puede afirmar que existe una gran correlación entre las variables acta y examen (.74), lo cual es lógico, ya que una gran parte de la calificación del acta depende de la puntuación conseguida en el examen. De la misma forma, la relación entre las publicaciones y las notas de Facebook también presentan una correlación alta (.71), ya que estas contaban el .65 de la calificación de la interacción de Facebook.

El único caso donde se produce una correlación inversa es entre el examen y las prácticas (-.21), ya que el primero es un instrumento de evaluación sumativa, mientras que las prácticas se definen como un instrumento de evaluación formativa; asimismo, el 75\% de las actividades prácticas son grupales, y lógicamente el examen tiene un carácter individual. Por lo tanto, ambos contribuyen al acta, en el caso de las prácticas se tiene un efecto mediano (.32) y en el del examen un efecto grande (.74).

Por otro lado, también son varias las relaciones que tienen un efecto pequeño. Concretamente, entre la calificación final en el acta y los comentarios en Facebook (.09), entre las calificaciones del acta y publicaciones en Facebook (.13), entre el examen y la nota Facebook (.14), y entre las prácticas y la nota Facebook (.14). Quedando este hecho justificado por la misma razón que se ha explicitado en el párrafo anterior. En esta ocasión, no se extrae una correlación inversa, puesto que la nota de Facebook ponderó menos que la de prácticas, sin embargo, sí que se obtiene una relación de variables muy cercana al valor 0 . Por lo que igualmente, presenta un efecto despreciable.

Por último, en relación a la principal pregunta de investigación del presente estudio se detecta una correlación media (.34) entre la nota final de la participación en Facebook y las calificaciones del acta final de la asignatura. Este hecho pone de manifiesto que la interacción en Facebook y la calificación final de la asignatura están relacionadas, pudiendo llegar a afirmar que los alumnos que han participado más en Facebook (tanto con publicaciones como con comentarios) son también los que más calificación han obtenido en la asignatura.

\section{Discusión y resultados}

En líneas generales, cabe destacar que debido al uso de Facebook se ha profundizado en determinados aspectos de la asignatura que, en el contexto presencial, por la limitación temporal, no hubiera sido posible. De esta manera, cabe rechazar la perspectiva de esta red social como una herramienta de ocio que interrumpe la tarea académica (Vivar-Zurita, et al., 2011), pues integrada como una herramienta complementaria tiene un elevado potencial educativo.

Los resultados evidencian la correlación positiva que se produce entre las calificaciones finales de la asignatura y la calificación de la interacción en Facebook. Por tanto, podemos concluir que, generalmente, los alumnos con una mayor participación en Facebook son los que más calificación han obtenido en la asignatura, aunque el grupo 3 difiere en este aspecto, principalmente, porque Facebook fue integrado en menor medida respecto a los otros grupos, ya que las directrices, el seguimiento y la implicación por parte del profesorado no fue el mismo. Teóricamente, este hecho, quedaría justificado debido a que el aprendizaje no se encuentra en función del medio -en este caso Facebook-, sino en función de la estrategia didáctica usada por el docente (Cabero, 2004). Recordemos que, como dice Prendes (2018), "la innovación educativa afecta a estrategias, procedimientos, recursos, metodologías, organización,... todos los elementos que conforman el sistema complejo de las organizaciones e instituciones educativas en el complejo cosmos de sus sistemas sociales" (p. 10). Por tanto, presuponer que la simple incorporación de Facebook 
conllevará una innovación educativa y una mejora en el proceso de enseñanza-aprendizaje será un gran error.

Por el tipo de evaluación planteada en la asignatura, de corte teórico-práctico y un elevado componente reflexivo frente al meramente conceptual, es de suponer que los alumnos que han obtenido mayor calificación en la asignatura tienen un nivel más comprensivo de la asignatura, y en ello ha podido contribuir la participación activa que este alumnado ha tenido en la red social y la interacción con el resto del alumnado y con el profesorado. Por tanto, en posteriores experiencias, habría que estar especialmente atento al alumnado que participa poco o nada, y plantear estrategias que promuevan la participación de todo el alumnado.

Para garantizar una buena práctica, fue necesario planificar la propuesta y consensuarla con los estudiantes previamente a implementarla, como establece López de la Madrid et al. (2017), ya que no tenían experiencia previa, y exceptuando algunos casos, como la inexistencia de comentarios o publicaciones en los 3 grupos por parte de algunos estudiantes, la calificación del acta se ha visto beneficiada con la participación en Facebook. Igualmente, y aunque el análisis no se ha focalizado en una perspectiva cualitativa, la interacción que se ha producido reproduce las ventajas de algunas propuestas ya implementadas: se ha favorecido el interés por los contenidos tratados en las sesiones presenciales (Viviar-Zurita, et al., 2011); desarrollo del pensamiento crítico (Castro \& González-Palta, 2016), debido al feedback que se generaba en los comentarios de algunas entradas, como por ejemplo en el caso de relacionar el sistema educativo de un país y su posicionamiento en el informe PISA; fortalecimiento del trabajo colaborativo (Abúndez et al., 2015), entre otras.

Tal y como se indicaba al inicio de este trabajo, la Tecnología Educativa está suponiendo un desafío para cualquier nivel educativo (Gamito et al., 2019). Concretamente, la implementación de Facebook como herramienta digital complementaria ha supuesto un esfuerzo de formación tanto por parte del alumnado, como del profesorado, lo cual justifica la necesidad mostrada por López de la Madrid et al. (2015), es decir, una formación específica para el profesorado sobre cómo utilizar didácticamente esta red social.

Como visión prospectiva de investigación se sugiere: completar las conclusiones de este estudio con una perspectiva cualitativa para analizar otras variables no tenidas en cuenta en este estudio (experiencia previa, satisfacción de uso, tiempo invertido, tipo de información compartida, entre otras); contemplar una perspectiva profesional a partir de la planificación, uso y evaluación realizada por los docentes implicados; establecer un grupo control para analizar las diferencias cuantitativas y cualitativas que se puedan generar; y por último, hacer uso de las emergentes análiticas de aprendizaje social (Social Learning Analytics) para estudiar problemas similares a los planteados en este trabajo.

Hay que destacar que en muy poco tiempo hemos pasado por grandes cambios en la docencia universitaria, y uno de los principales es que «las redes no suponen únicamente un nuevo modelo de comunicarse, sino que además se convierten en un nuevo modo de construcción compartida del conocimiento» (Martínez \& Prendes, 2003, p. 44). En este sentido, Area (2018) se plantea que esta «universidad digital» tiene sus luces y sombras, ya que, aunque existe aún una resistencia a superar el paradigma pedagógico tradicional, cada vez hay más docentes e instituciones que realizan experiencias de innovación que parten del aprendizaje activo y que se apoyan en las TIC para construir, compartir y evaluar estas experiencias. 


\section{Referencias}

Abúndez, E., Fernández, F., Meza, L.E. y Alamo, M.C. (2015). Facebook como herramienta educativa en el proceso de enseñanza-aprendizaje en el nivel medio superior. Zona próxima, 22, 116-127. https:// doi.org/10.14482/zp.22.6330

Adams, B., Raes, A., Montrieux, H. y Schellens, T. (2018). "Pedagogical tweeting" in higher education: boon or bane? International Journal of Educational Technology in Higher Education, 15(1). https://doi.org/ $10.1186 / \mathrm{s} 41239-018-0102-5$

Alvarado, H. y Batanero, C. (2008). Significado del teorema central del límite en textos universitarios de probabilidad y estadística. Estudios pedagógicos, 34(2), 7-28. http://bit.ly/36ekjzU

Area, M. (2018). Hacia la Universidad digital: ¿Dónde estamos y a dónde vamos? RIED. Revista Iberoamericana de Educación a Distancia. 21(2), 25-30. http://dx.doi.org/10.5944/ried.21.2.21801

Arteaga, R., Cortijo, V. \& Javed, U. (2014). Students'perceptions of Facebook for academic purposes. Computers \& Education, 70, 138-149. https://doi.org/10.1016/j.compedu.2013.08.012

Arteaga-Sánchez, R., Cortijo, V. \& Javed, U. (2019). Factors driving the adoption of Facebook in higher education. E-learning and digital media, 16 (6), 455-474. https://doi.org/10.1177/2042753019863832

Cabero, J. (2004). No todo es Internet: Los medios audiovisuales e informáticos como recursos didácticos. Comunicación y pedagogía, 200, 19-23.

Cabero, J. y Agauded, J. (2007). Avances y retos en la promoción de la innovación didáctica con las tecnologías emergentes e interactivas. Revista Educar, especial 30 aniversario, 67-83. http://bit.ly/2vYtb00

Cabero, J., Llorente, M.C. y Gisbert, M. (2007). El papel del profesor y el alumno en los nuevos entornos tecnológicos de formación. En Julio Cabero (Ed.). Nuevas tecnologías aplicadas a la educación. McGraw-Hill. https://bit.ly/2nPdLqS

Cabeza, L., González, N. y Martín, R. (2014). Facebook como herramienta para motivar a los alumnos universitarios. En M.T. Ramiro, T. Ramiro y M.P. Bermúdez (Eds.), FECIES 2013 (pp. 950-954). Asociación Española de Psicología Conductual. https://bit.ly/3e7HVdI

Calzado, M.A. et al. (2018). El uso de redes sociales como instrumento innovador en la educación universitaria: Facebook como herramienta de aprendizaje colaborativo en la asignatura de inmunología. Revista de innovación y buenas prácticas docentes, 4, 32-48. http://bit.ly/31ESoZ4

Chugh, R., Ruhi, U. Social media in higher education: A literature review of Facebook. Educ Inf Technol 23, 605-616 (2018). https://doi.org/10.1007/s10639-017-9621-2

Cornejo, J.F. y Lariza, K. (2016). Impacto de las redes sociales en los procesos de enseñanza-aprendizaje de educación superior pública, boletín Redipe, 5(11), 154-175. https://bit.ly/2nNXqTA

Cunha, F.R., Van Kruistum, C. \& Van Oers, B. (2016). Teachers and Facebook: using online groups to improve student's communication and engagement in education. Communication Teacher, 30(4), 228-241. https://doi.org/10.1080/17404622.2016.1219039

Davidovitch, N. \& Belichenko, M. (2018). Facebook Tools and Digital Learning Achievements in Higher Education. Journal of Education and e-Learning Research, 5 (1), 8-14, https://doi.org/10.20448/ journal.509.2018.51.8.14 F

De Haro, J.J. (2009). Las redes sociales aplicadas a la práctica docente. Didáctica, Innovación y Multimedia, 13. https://bit.ly/2nOnpKC

Díaz-Lázaro, J.J., Solano-Fernández, I.M. y Sánchez-Vera, M.M. (2017). Social learning analytics in higher education. An experienceat the primary education stage. Journal of New Approaches in Educational Research, 6(2), 119-126. https://doi.org/10.7821/naer.2017.7.232

Gamito, R., Aristizabal, P. y Vizcarra, M.T. (2019). Sociedad multipantalla: un reto educativo para familia y escuela. Prisma Social: revista de investigación social, 25, 398-423. https://bit.ly/2mkDcjS 
González-Pérez, A. (2018). Social networks as tools to enrich learning environments in higher education. Bordón. Revista de pedagogía, 70 (4), 55-71. https://doi.org/10.13042/Bordon.2018.60579

Gutiérrez-Porlán, I., Román-García, M. y Sánchez-Vera, M. (2018). Estrategias para la comunicación y el trabajo colaborativo en red de los estudiantes universitarios. Comunicar, 54, 91-100. https://doi.org/10.3916/ C54-2018-09

Iglesias-García, M. y González, C. (2014). Facebook como herramienta educativa en el contexto universitario. Historia y comunicación social, 19(1), 379-391. https://doi.org/10.5209/rev_hics.2014.v19.45606

Imbernón, F. (2016). Los retos educativos del presenre y del futuro. La sociedad cambia, ¿y el profesorado? Revista Internacional de Formação de Professores, 1(1), 121-129. http://bit.ly/3aVcXUA

López-Soto, P.J. (2013). Contraste de hipótesis. Comparación de más de dos medias independientes mediante pruebas no paramétricas: prueba de Kruskal-Wallis. Revista Enfermería del Trabajo, 3(4), 166-171. https://bit.ly/3e2VEmy

López de la Madrid, M.C., Flores, K. y Espinoza, A. (2015). Diversidad de usos de Facebook en la educación superior. Análisis desde un caso de estudio. Innoeduca, 1(2), 106-114. https://doi.org/10.20548/ innoeduca.2015.v1i2.1040

López de la Madrid, M.C., Flores, K., Espinoza, A. y Rojo, D. (2017). Posibilidades de Facebook en la docencia universitaria desde un caso de estudio. Apertura, 9(2), 132-147. https://bit.ly/3bNIoAt

Lytras, M. D., Visvizi, A., Sarirete, A. \& Ordonez-De Pablos, P. (2018). Social Networks Research for Sustainable Smart Education. Sustainability 10 (9), 2974. https://doi.org/10.3390/su10092974

Marín-Diaz, V., y Cabero-Almenara, J. (2019). Las redes sociales en educación: desde la innovación a la investigación educativa? RIED. Revista Iberoamericana de Educación a Distancia, 22(2), pp. 25-33. http://dx.doi.org/10.5944/ ried.22.2.24248

Manca, S. (2020). Snapping, pinning, liking or texting: Investigating social media in higher education beyond Facebook. The Internet and Higher Education, 44, sin paginar (preprint). https://doi.org/10.1016/ j.iheduc.2019.100707

Martínez, F. y Prendes, M.P. (2004). Nuevas Tecnologías y Educación. Pearson.

Martínez, M.C. y Ferraz, E. (2016). Uso de las redes sociales por los alumnos universitarios de educación: un estudio de caso de la península ibérica. Tendencias educativas, 28. http://bit.ly/2HkYGUJ

Mateo, J. (2004). La investigación ex post-facto. En R. Bisquerra (Ed.), Metodología de la investigación educativa (pp. 187-222). La Muralla.

Nicolai, L., et al. (2017). Facebook groups as a powerful and dynamic tool in medical education: mixed-method study. Journal of Medical Internet Research, 19(12). https://doi.org/10.2196/jmir.7990

Prendes, M. P. (2018). La Tecnología Educativa en la Pedagogía del siglo XXI: una visión en 3D. Revista Interuniversitaria De Investigación En Tecnología Educativa, (4), 6-16. https://doi.org/10.6018/riite/ $2018 / 335131$.

Prendes, M.P. y Román, M.M. (2017). Entornos Personales de Aprendizaje. Octaedro.

Prensky, M. (2001). Nativos e inmigrantes digitales. España, Madrid: Distribuidora SEK. https://bit.ly/2w6wfW6

Prensky, M., y Gonzalez Calatayud, V. (2018). Entrevista a Marc Prensky. Revista Interuniversitaria De Investigación En Tecnología Educativa, (5), 12-21. https://doi.org/10.6018/riite/2018/354791

Rocha, M. A., Amador, G. y Álvarez, I. B. (2015). Las redes sociales: el acompañamiento emocional de los estudiantes durante la movilidad estudiantil universitaria. Revista Electrónica de Tecnología Educativa, 53, 1-17. https://bit.ly/2oA8ion

Serrat, N. (2015). Metodologías participativas y Facebook en el ámbito universitario. Innoeduca. International journal of technology and educational innovation, 1(1), 25-32. http://bit.ly/2OGFMeN 
Shraim, K. (2014). Pedagogical innovation within Facebook: a case study in tertiary education in Palestine. International Journal of Emerging Technologies in Learning, 9(8), 25-31. https://doi.org/10.3991/ ijet.v9i8.3805

Siemens, G. y Gasevic, D. (2012). Learning and Knowledge Analytics. Educational Technology \& Society 15(3), 1-2. https://bit.ly/2MhyrSb

Thai, M. , Sheeran, N. \& Cummings, D.J. (2019). We 're all in this together: The impact of Facebook groups on social connectedness and other outcomes in higher education. The Internet and Higher Education, 40, 44-49. https://doi.org/10.1016/j.iheduc.2018.10.001

Tobón, C.A. y Bedoya, J.R. (2017). Influencia de la asimetría en el tamaño de la muestra para el cumplimiento del teorema central del límite en distribuciones continúas. Scientia et Technica, 22(4), 398-402. https://bit.ly/2YJZ9Yy

Túñez López, M., Valdiviezo Abad, C. y Martínez Solana, C. (2015). Las redes sociales en la gestión de la comunicación universitaria. Opción, 31(6), 852-874. https://bit.ly/2mdXjQw

Uğur, N.G. y Koç., T. (2019). Leading and Teaching with Technology: School Principals' Perspective. International Journal of Educational Leadership and Management, 7(1), 42-71. https://doi.org/ 10.17583/ijelm.2019.3758

Vivar-Zurita, H., García-García, A., Abuín-Vences, N., Vinader-Segura, R., Núñez-Gómez, P. y Martín-Pérez, M.A. (2011). La innovación educativa en la enseñanza superior: Facebook como herramienta docente. Revista de Comunicación Vivat Academia, 117, 530-544. https://doi.org/10.15178/ va.2011.117e.530-544

White, D. (2011). Not natives or immigrants, but visitors and residents. http://bit.ly/2vktPEI

White, D. y Le Cornu, A. (2011). Visitors and residents: a new typology for online engagement. First Monday, 16(9). https://doi.org/10.5210/fm.v16i9.3171

Zanon, M., Altmayer, S., Sartori, G., Guedes, A., Watte, G. Marchiori, E. y Hochhegger, B. (2018). Facebook as a tool to promote radiology education: expanding from a local community of medical students to all of South America. Radiologia Brasileira, 51(4). https://doi.org/10.1590/0100-3984.2017.0112

Zuñe, L. y Rosas, C.E. (2016). Facebook como herramienta pedagógica en los procesos de enseñanza aprendizaje. UCV HACER, 5(1), 24-32. https://doi.org/10.18050/ucv-hacer.v5i1.957 